\title{
Research on Bi-level Cooperative Robust Planning of Distributed Renewable Energy in Incremental Distribution Network Considering Demand Response
}

\author{
Sui $\mathrm{Yi}^{1 \mathrm{a}^{*}}$, Wu Jianbin ${ }^{1}$, Liu Huiqing ${ }^{1}$, Li Chunhui ${ }^{1}$, Zhao Shuai ${ }^{1}$, Gao Jin $^{1}$, Bai Yang ${ }^{1}$ \\ ${ }^{1}$ State Grid Shanxi Electric Power Company Economic and Technical Research Institute, Taiyuan, Shanxi
}

\begin{abstract}
The paper constructs a two-level collaborative planning model for incremental distribution network considering demand response and distributed renewable energy access. In the upper model, the goal is to minimize the investment cost of the distribution network, and the lower model takes the system's operating cost optimally, fully considers the uncertainty of renewable energy output, and introduces robust optimization to solve it. It can be seen from the simulation results that the consideration of demand response in the distribution network planning is conducive to delaying investment costs, enhancing power user load flexibility, and effectively avoiding load shedding and other problems. The research results lay the foundation for the feasibility of demand response resources participating in power grid planning
\end{abstract}

\section{1 introduction}

global climate change and the increasing shortage of fossil energy resources have made society pay more and more attention to energy and environmental issues. "Doing a good job in carbon peaking and carbon neutrality" has also been listed as one of China's key tasks. Therefore, it has become an inevitable trend for distributed renewable energy with high penetration rate to be integrated into the incremental distribution network [1-3]. The rapid development of distributed renewable energy not only brings opportunities for energy structure transformation, but also poses new challenges to the power grid, such as changes in the topology of the distribution network [4-5].

Regarding the planning of distributed renewable energy in the incremental distribution network, relevant scholars at home and abroad have carried out relevant research. At present, the main research methods are economic planning model and planning-operation twolevel model. In the incremental distribution network planning, if only focusing on the planning of distributed renewable energy, the planning may be too simple and unable to make full use of other resources in the distribution network. Nowadays, demand response plays an increasingly important role in incremental distribution network planning. This approach has changed the thinking that power sources passively accept user load changes [6-7],

This paper takes into account the energy consumption problem of distributed renewable energy connected to the active distribution network and the impact of demand response on the operation of the distribution network, and proposes a new incremental distribution network planning method.

\section{Cooperative Planning Model of Incremental Distribution Network}

\subsection{Upper-level planning model}

The decision variables of the upper-level planning are the location and capacity of distributed renewable energy access, the purchase capacity and location of demand response; the objective function is the total cost of the plan, including the investment cost of distributed renewable energy, the capacity cost of demand response, and The operating cost of the system,etc.

\subsubsection{Objective function}

$$
\begin{gathered}
\min F_{T}=C_{i n v}+w C_{o p e} \\
C_{i n v}=k_{\text {res }} \sum_{i \in N_{\text {res }}} c_{\text {res }} n_{i, r e s}+\sum_{i \in N_{D R}} c_{R D} n_{R D, i} \\
\kappa_{\text {res }}=\frac{a(1+a)^{b}}{(1+a)^{b}-1}
\end{gathered}
$$

In the formula, $C_{i n v}$ represents the planning cost of distributed power; $C_{\text {ope }}$ represents the operating cost of the system, which is calculated and transmitted by the underlying model; $w$ represents the conversion factor of the net present value of the operating layer. $k_{r e s}$ represents the annual investment equivalent coefficient of renewable energy; a represents the discount rate; $b$ represents the average service life of renewable energy power generation equipment; ${ }^{c_{\text {res }}}$ represents the unit investment cost of renewable energy equipment; ${ }^{n_{i, e s}}$ represents the

\footnotetext{
a Sui Yi: 2677016110@qq.com
} 
number of renewable energy equipment at the i-th node; $c_{R D}$ represents the unit cost of demand response capacity; $n_{R D, i}$ represents the demand response capacity;

\subsubsection{Restrictions}

Due to the limitations of space and funds, there are also certain restrictions on the number of devices that can be installed on each node in the newly added distribution network. which is:

$$
0 \leq n_{i, r e s} \leq N_{i, r e s}
$$

In the formula, $N_{i, r e s}$ represents the upper limit of the number of renewable energy equipment at the i-th node.

$$
S_{D R, i}^{\min } \leq S_{D R, i} \leq S_{D R, i}^{\max }
$$

In the formula, $S_{D R, i}^{\min } 、 S_{D R, i}^{\max }$ respectively represent the upper and lower limits of the demand response capacity that can be purchased at node $i$.

\subsection{Low-level robust optimization model}

The lower-level optimization is the optimal dispatch situation of demand response on a typical day of wind power. The decision volume is the output of demand response and the $0-1$ variables that determine whether to call demand response at this moment, the amount of air abandonment per hour and the amount of load shedding; the objective function is the operating cost of the entire system, including the power purchase cost of the system excluding network loss, Operation and maintenance costs of renewable energy equipment, electricity costs for demand response, wind abandonment costs, and load shedding costs. Constraints include demand response constraints, power balance constraints, and so on..

\subsubsection{Objective function}

$$
\begin{aligned}
& C_{\text {ope }}=C_{\text {grid }}+C_{\text {opr }}^{r e s}+C_{r e s}^{c}+C_{\text {load }}^{c}+C_{o p r}^{D R} \\
& C_{\text {grid }}=p_{\text {tou }} \sum_{t \in T} P_{\text {grid }} \\
& C_{o p r}^{r e s}=\sum_{t \in T} \sum_{i \in N_{r e s}}\left(c_{w}^{o p r} P_{i, t, w}+c_{p v}^{o p r} P_{i, t, p v}\right) \\
& C_{\text {res }}^{c}=\sum_{t \in T} \sum_{i \in N_{\text {res }}} c_{\text {res }}^{c} c_{u t}^{\text {res }} \\
& C_{\text {load }}^{c}=\sum_{t \in T} \sum_{i \in N_{l}} c_{\text {load }}^{c} c u t_{i, t}^{\text {load }} \\
& C_{o p r}^{D R}=\sum_{t \in T} \sum_{i \in N_{D R}} c_{o p r}^{D R}\left(D R_{i, t}^{+}+D R_{i, t}^{-}\right)
\end{aligned}
$$

In the formula, $C_{\text {grid }}, C_{\text {opr }}^{\text {res }}, C_{\text {res }}^{c}, C_{\text {load }}^{c}, C_{\text {opr }}^{D R}$ are the cost of purchasing electricity from the main grid, the operating cost of renewable energy, the penalty cost of renewable energy curtailment, the cost of load shedding, and the demand response operation. cost. $p_{\text {tou }}, c_{\text {res }}^{o p r}$, $c_{\text {res }}^{c}, \quad c_{\text {load }}^{c}, \quad c_{\text {opr }}^{D R}$ respectively represent the time-of-use electricity price of the main network, the unit electricity cost of renewable energy, the unit penalty cost of abandonment, the penalty cost of load shedding, and the demand response unit Electricity cost. $L_{i, t}$ represents the load demand of the $\mathrm{i}$-th node at time $\mathrm{t} ; \mathrm{cut}_{i, t}^{\text {res }}, \mathrm{cut}_{i, t}^{\text {load }}$ respectively represent the load demand of the $i$-th node at time $t$ The amount of power discarded and load shedding of i nodes. $D R_{i t}^{+}$and $D R_{i t}^{-}$respectively represent the peak shaving volume and valley filling volume of demand response at time $\mathrm{t}$; $P_{\text {grid }}$ refers to the purchase of electricity from the main network.

\subsubsection{Restriction}

The lower-level optimization is the optimal dispatch situation of demand response on a typical day of wind power. The decision volume is the output of demand response and the $0-1$ variables that determine whether to call demand response at this moment, the amount of air abandonment per hour and the amount of load shedding; the objective function is the operating cost of the entire system, including the power purchase cost of the system excluding network loss, Operation and maintenance costs of renewable energy equipment, electricity costs for demand response, wind abandonment costs, and load shedding costs. Constraints include demand response constraints, power balance constraints, and so on.

1) Power balance constraint:

$$
\begin{aligned}
& P_{\text {grid }, t}+\sum_{i \in N_{\text {res }}} P_{i, t}^{\text {res }}-\sum_{i \in N_{\text {res }}} c u t_{i, t}^{\text {res }}+ \\
& \sum_{i \in N_{l}} c u t_{i, t}^{\text {load }}+\sum_{i \in N_{D R}}\left|D R_{i, t}^{+}-D R_{i, t}^{-}\right| \\
& =\sum_{i \in N} L_{i, t}, \forall i, t
\end{aligned}
$$

2) DR constraint:

$$
\begin{gathered}
0 \leq D R_{i, t}^{+} \leq u_{i, t}^{+} S_{D R, i}^{\max }, \forall i, t \\
0 \leq D R_{i, t}^{-} \leq u_{i, t}^{-} S_{D R, i}^{\max }, \forall i, t \\
u_{i, t}=u_{i, t}^{+}+u_{i, t}^{-}, \forall i, t \\
0 \leq u_{i, t}^{+}+u_{i, t}^{-} \leq 1, \forall i, t \\
c_{o p r}^{D R}=\left\{\begin{array}{c}
C_{1 i}, 0<\left|D R_{i, t}^{+}-D R_{i, t}^{-}\right| \leq \frac{1}{3} S_{D R, i}^{\max }, \forall i, t \\
C_{2 i}, \frac{1}{3} S_{D R, i}^{\max }<\left|D R_{i, t}^{+}-D R_{i, t}^{-}\right| \leq \frac{2}{3} S_{D R, i}^{\max }, \forall i, t \\
C_{3 i}, \frac{2}{3} S_{D R, i}^{\max }<\left|D R_{i, t}^{+}-D R_{i, t}^{-}\right| \leq S_{D R, i}^{\max }, \forall i, t
\end{array}\right.
\end{gathered}
$$

$3)$ Renewable energy constraints:

$$
0 \leq P_{i, t, r e s} \leq P_{r e s}^{\max }, \forall i, t
$$

4) Mainnet purchase capacity constraints:

$$
0 \leq P_{\text {grid }, t} \leq P_{\text {grid }}^{\max }, \forall t
$$

5) Load shedding constraint:

$$
0 \leq c u t_{i, t}^{\text {load }} \leq c u t_{i, t}^{\text {load,max }}, \forall i, t
$$

6 ) Wind abandonment and solar abandonment constraints :

$$
0 \leq c u t_{i, t}^{r e s} \leq c u t_{i, t}^{r e s, \max }, \forall i, t
$$

7) Robustness constraints

$$
\begin{gathered}
H:=\left\{P_{G, R}=\left(P_{\text {grid }, t}, P_{i, t, \text { res }}\right):\right. \\
\hat{P}_{\text {res }} \in U, \exists \Delta P_{\text {grid }}=\left(P_{\text {grid }}^{1}, P_{\text {grid }}^{2}, \cdots, P_{\text {grid }}^{N_{\text {res }}}\right)
\end{gathered}
$$




$$
\begin{aligned}
& \Delta P_{\text {grid }, t}+\sum_{i \in N_{\text {res }}}\left(\hat{P}_{i, t}^{r e s}-P_{i, t}^{r e s}-c u t_{i, t}^{r e s}\right)+ \\
& \sum_{i \in N_{l}} \Delta c u t_{i, t}^{\text {load }}=\sum_{i \in N_{D R}} \Delta\left|D R_{i, t}^{+}-D R_{i, t}^{-}\right|, \forall i, t \\
& 0 \leq P_{\text {grid,t }}+\Delta P_{\text {grid, } t} \leq P_{\text {grid }}^{\max }, \forall t \\
& 0 \leq \mathrm{cut}_{i, t}^{r e s}+\Delta c u t_{i, t}^{r e s} \leq \mathrm{cut} t_{i, t}^{\text {res,max }}, \forall i, t
\end{aligned}
$$

$$
\left.0 \leq c u t_{i, t}^{\text {load }}+\Delta c u t_{i, t}^{\text {load }} \leq c u t_{i, t}^{\text {load,max }}, \forall i, t\right\}
$$

In the formula, $P_{i, t}^{r e s}$ refers to the amount of electricity generated by renewable energy; $\mathrm{cut}_{i, t}^{\text {res }}$ refers to the amount of electricity discarded from renewable energy; $c u t_{i, t}^{\text {load }}$ refers to the load shedding in the system; $L_{i, t}$ is the system load. $u_{i, t}^{+}$and $u_{i, t}^{-}$are $0-1$ variables; $C_{1 i}$ 、 $C_{1 i}, C_{1 i}$ are the unit electricity cost involved in demand response, respectively. $\Delta P_{\text {grid }}$ is the adjustment variable for the amount purchased from the main network; $\Delta c u t_{i, t}^{\text {load }}$ refers to the adjustment variable for load shedding; $\Delta\left|D R_{i, t}^{+}-D R_{i, t}^{-}\right|$represents the adjustment variable of demand response. Equations (23)-(28) express the robustness constraints, that is, when the actual wind power output $\hat{P}_{\text {res }}$ deviates from the predicted value $P_{\text {res }}$, the system scheduling rebalancing is achieved through equation (25), equations (26)-( 28) Indicates that its variables should still meet its corresponding constraints during rescheduling..

\section{Collaborative programming model solving method}

From the description of the model above, we can see that the upper-level programming model constructed in this article is a simple linear programming problem, which can be solved directly using CPLEX optimization software. Since the lower-level optimization model contains renewable energy that cannot accurately predict the output, the model cannot be solved directly. The commonly used uncertainty optimization processing methods are stochastic optimization and robust optimization. This article will use robust optimization to deal with them. The specific expression is as follows:

$(M P) \cdot \min C_{\text {ope }}=C_{\text {grid }}+C_{\text {opr }}^{\text {res }}+C_{\text {res }}^{c}+C_{\text {load }}^{c}+C_{\text {opr }}^{D R}$

s.t.

$$
\text { (12)-(22) }
$$

$\Delta p_{\text {grid }}^{k, t}+\sum_{i \in N_{\text {res }}}\left(P_{i, t, k}^{\hat{\text { res }}}-p_{i, t}^{\text {res }}-c u t_{i, t}^{\text {res }}\right)+\sum_{i \in N_{L}} \Delta c u t_{i, t, k}^{\text {load }}$

$=\sum_{i \in N_{D R}} \Delta\left|D R_{i, t, k}^{+}-D R_{i, t, k}^{+}\right|, \forall i, t, k$

$$
0 \leq P_{\text {grid, } t}+\Delta P_{\text {grid }}^{k, t} \leq P_{\text {grid }}^{\max }, \forall t, k
$$

and:

$$
(S P) \mathbb{Z}=\max _{P_{\text {res }} \in U} \min _{S^{+}, s^{-}, \Delta P \text { grid }} \sum_{i \in N} \sum_{t \in T}\left(s_{i, t}^{+}+s_{i, t}^{-}\right)
$$

s.t.

$$
\begin{gathered}
\Delta p_{\text {grid }}^{k, t}+\sum_{i \in N_{\text {res }}}\left(P_{i, t, k}^{r e s}-p_{i, t}^{r e s}-c u t_{i, t}^{\text {res }}\right)+\sum_{i \in N}\left(s_{i, t}^{+}+s_{i, t}^{-}\right) \\
+\sum_{i \in N_{l}} \Delta c u t_{i, t}^{\text {load }}=\sum_{i \in N_{D R}} \Delta\left|D R_{i, t}^{+}-D R_{i, t}^{-}\right|, \forall i, t \\
s_{i, t}^{+} \geq 0, \forall i, t \\
s_{i, t}^{-} \geq 0, \forall i, t \\
(26)-(28)
\end{gathered}
$$

In the formula: $\kappa(\mathrm{k} \in \kappa)$ is the set of uncertainty factors $P_{\text {res }}$, which gradually expands with the iterative process of solving the sub-problems. In the objective function of the sub-problem, $s_{\mathrm{n}, t}^{+} 、 \mathrm{~s}_{n, t}^{-}$is a non-negative slack variable, which can be used to evaluate the improper correlation in solving the main problem, and it can also represent uncertain factors such as load shedding due to system constraints. Therefore, to solve the sub-problem is to find the worst point $P_{\text {res, } k}$ in the uncertainty set $\mathrm{u}$, and obtain the economic dispatch plan accordingly. The calculation process is as follows:

1) $\mathrm{K} \leftarrow \varnothing, k \leftarrow 1, \mathbb{Z} \leftarrow+\infty$, And define its error tolerance as $\delta$;

2) When $Z \geq \delta$, enter the next step of calculation;

3) Solve the main problem (MP) and get the optimal solution;

4) Substitute the optimal solution obtained in the previous step into the sub-problem (SP), and solve (SP) to obtain the result $\left(\mathrm{Z},\left(\mathbb{Z}, \widehat{W_{k}}\right)\right)$;

5) $k \leftarrow k \cup K, k \leftarrow k+1$;

6) When the conditions are met, the loop ends.

\section{Simulation calculation}

\subsection{Calculation example environment and parameters}

This text carries on the simulation analysis of the distribution network planning based on the IEEE 33-node system, as shown in Figure 1. The unit installed capacity cost of wind turbines is $1.19 \times 10^{\wedge} 7$ yuan $/ \mathrm{MW}$, and the unit installed capacity cost of photovoltaics is $2.19 \times 10^{\wedge} 7$ yuan/MW. In addition, the maximum number of connected positions of the wind turbine is 5 , and the candidate nodes are $3,8,10,23$, and 30 . The maximum number of $\mathrm{PV}$ access locations is also 5, and the candidate nodes are 4, 9, 19, 26, and 32. In addition, the maximum connection capacity of a single node for wind turbines and photovoltaics is $1 \mathrm{MW}$. The output range of wind and wind is shown in Figure 2 and Figure 3.

Demand response can participate in both the capacity market and the electric energy market. Based on this, this article first assumes that the load nodes that can participate in demand response are 3, 9, 19, 23, and 26. Secondly, the load change of each load node after the implementation of demand response is 0.8 to 1.4 times the original load.

The time-of-use electricity price is shown in Table 1. In order to promote the full utilization of wind power and photovoltaic resources and suppress load fluctuations, this 
paper sets the cost of abandoning wind and solar power and the cost of load shedding to 1 yuan/(MW'h), and the planned period is $10 \mathrm{a}$.

Table1. Peak valley TOU price

\begin{tabular}{|c|c|c|}
\hline Time & Specific moment & price $(\mathrm{yuan} / \mathrm{kW} \mathrm{h})$ \\
\hline $\begin{array}{c}\text { Peak } \\
\text { time }\end{array}$ & $08: 00-11: 00 ; 17: 00-22: 00$ & 0.5264 \\
\hline $\begin{array}{c}\text { Normal } \\
\text { time }\end{array}$ & $06: 00-08: 00 ; 11: 00-17: 00$ & 0.4028 \\
\hline $\begin{array}{c}\text { Valley } \\
\text { time }\end{array}$ & $00: 00-06: 00 ; 22: 00-24: 00$ & 0.3256 \\
\hline
\end{tabular}

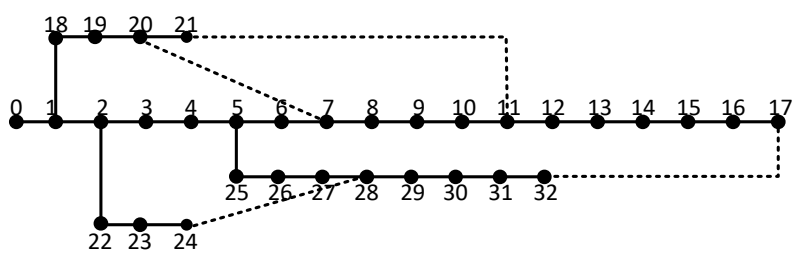

Fig 1. Wiring diagram of IEEE33 node power distribution system

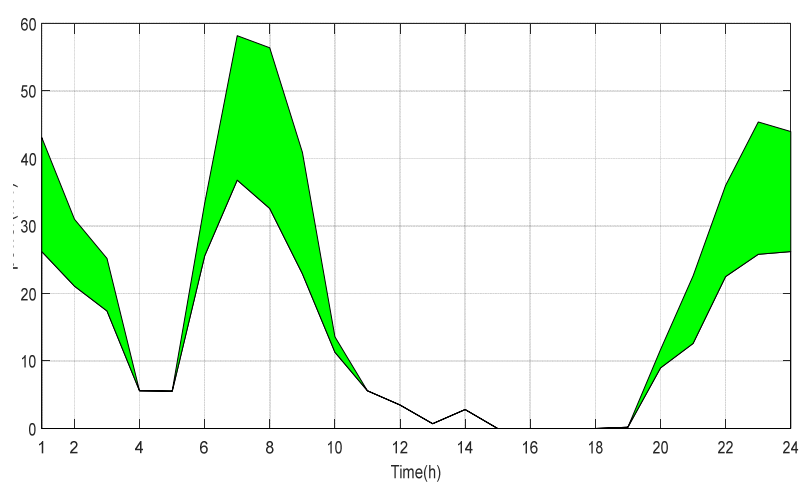

Fig 2. Wind power output range

\subsection{Impact analysis of demand response}

\subsubsection{The impact of demand response on planning results}

In the incremental distribution network planning, the inclusion of a demand response mechanism will have a certain impact on its planning. In order to quantify the impact of the demand response mechanism, this article will set up two scenarios:

Scenario 1: Demand response mechanism is not considered in the incremental distribution network planning of renewable energy;

Scenario 2: Consider the demand response mechanism in the incremental distribution network planning of renewable energy.

Table2. Comparison of planning results between scenario 1 and scenario 2

\begin{tabular}{cccc}
\hline \multicolumn{2}{c}{ Scenario 1 } & \multicolumn{2}{c}{ Scenario 2 } \\
\hline position & capacity & position & capacity \\
3 & 0.70 & 3 & 0.65 \\
8 & 0 & 8 & 0 \\
\hline
\end{tabular}

\begin{tabular}{cccc}
\hline 10 & 0.85 & 10 & 0.70 \\
23 & 0.90 & 23 & 0.70 \\
30 & 0 & 30 & 0 \\
\hline
\end{tabular}

It can be seen from the configuration capacity that after considering the demand response in the system, the configuration capacity of renewable energy has decreased, which can improve the economy of the system while ensuring the reliability of the system.

\subsubsection{The impact of demand response on system costs}

The comparison results of system planning and operating costs in the two scenarios are shown in Table 3.

Table3. Cost comparison of different scenarios

\begin{tabular}{ccc}
\hline \multirow{2}{*}{ Cost item } & \multicolumn{2}{c}{$\begin{array}{c}\text { cost (Ten thousand } \\
\text { yuan ) }\end{array}$} \\
\cline { 2 - 3 } & Scenario 1 & Scenario 2 \\
\hline cost of investment & 67.34 & 39.53 \\
Renewable energy operating & 59.85 & 31.91 \\
costs & & \\
Main network power purchase & 524.76 & 509.37 \\
cost & 20.31 & 0.39 \\
Wind curtailment cost & 27.67 & 0 \\
Load shedding cost & 0 & 1.21 \\
DR Capacity cost & 0 & 8.92 \\
DR Electricity cost & 699.93 & 591.33 \\
total cost &
\end{tabular}

From the comparative analysis of the scenario's renewable energy operating costs, wind abandonment costs, and total costs, it can be found that demand response also has a greater effect on improving the consumption of renewable energy and reducing the phenomenon of wind and solar abandonment.

\subsection{Comparative analysis}

\subsubsection{Comparative analysis of planning models}

In order to further verify the superiority of the two-level collaborative planning model in this paper, the simulation results of this model will be compared with the singlelevel economic planning model and the simulation results of the single-level optimization operation.

Table4. Comparative simulation results of different models

\begin{tabular}{cccc}
\hline model & $\begin{array}{c}\text { cost of } \\
\text { investment }\end{array}$ & $\begin{array}{c}\text { Demand } \\
\text { response } \\
\text { capacity } \\
\text { cost }\end{array}$ & $\begin{array}{c}\text { Renewable } \\
\text { energy } \\
\text { abandonment } \\
\text { cost }\end{array}$ \\
\hline $\begin{array}{c}\text { Double } \\
\text { layer } \\
\text { Single } \\
\text { layer }\end{array}$ & 39.53 & 1.21 & 0.39 \\
\hline
\end{tabular}

It can be seen from Table 4 that the investment cost of the two-tier programming model is lower than that of the single-tier optimization model. The main reason is that the 
two-tier model takes the operating factors of the system into account during planning, so as to ensure that the system operates better. The configuration capacity of the equipment is conducive to reducing investment costs. In addition, the lower-level model in the two-level programming model constructed in this paper fully considers the uncertainty of renewable energy output, and uses robust optimization methods to optimize its operation, so as to reduce the abandonment of renewable energy as much as possible. Penalty costs.

\subsubsection{Comparative analysis of optimization algorithms}

The optimal investment cost obtained by SSA optimization algorithm is much lower than genetic algorithm and particle swarm algorithm. In addition, from the two dimensions of the number of iterations and iteration time, it can be seen that the SSA optimization algorithm is also lower than the genetic algorithm and the particle swarm algorithm, which proves that the global search capability and iteration efficiency of the SSA optimization algorithm are higher than the other two optimizations. algorithm.

Table5. Comparative analysis of different optimization algorithms

\begin{tabular}{cccc}
\hline algorithm & $\begin{array}{c}\text { cost of } \\
\text { investment }\end{array}$ & $\begin{array}{c}\text { Number of } \\
\text { iterations }\end{array}$ & $\begin{array}{c}\text { Iteration } \\
\text { time }\end{array}$ \\
\hline SSA & 39.53 & 321 & 276 \\
GA & 61.03 & 1230 & 1291 \\
PSA & 58.64 & 973 & 1527 \\
\hline
\end{tabular}

\section{Conclusion}

In the planning and research of the incremental distribution network, this paper further considers the impact of demand response resources and distributed renewable energy access, constructs a collaborative planning model of upper and lower levels, and uses renewable energy in the lower level planning model. Taking the uncertain factors into account, the robust optimization method is used to optimize the operating cost of the system, and returns to the upper-level planning model, so as to solve the optimal renewable energy equipment planning and configuration plan. Through simulation, the following conclusions can be drawn:

1) This model can effectively save investment costs (from 512,700 yuan to 395,300 yuan) when comprehensively considering investment and operating factors. In addition, the demand response factor is further introduced into the collaborative planning model, and the cost impact is considered from the two perspectives of demand response capacity cost and electricity cost. There are significant savings in investment costs, operating costs, load shedding costs, etc., so that the total cost of the system is reduced.

2) In the lower-level operation optimization model, a robust optimization model considering the uncertainty of renewable energy output is constructed. It can be seen from the results that by using the robust optimization model, the impact of uncertainty in the system on system operation can be better suppressed, and the amount of renewable energy discarded is greatly reduced, and the utilization efficiency of renewable energy is improved.

\section{References}

1. Azizivahed A, Arefi A, Ghavidel S, et al. Energy management strategy in dynamic distribution network reconfiguration considering renewable energy resources and storage[J]. IEEE Transactions on Sustainable Energy, 2019, 11(2): 662-673.

2. Dai Jie, Ma Zhao, Shang Yuwei, et al. (Dai Jie, Ma Zhao, Shang Yuwei, et al.). Overview of renewable distribution generation in UK and DNO progress (Overview of renewable distribution generation in UK and DNO progress). Advanced Technology of Electrical Engineering and Energy, 2017, 36(09): 43-50.

3. Lu Chang, Guo Li, Chai Yuanyuan et al. (Lu Chang, Guo Li, Chai Yuanyuan et al.). Day-ahead optimal scheduling method for incremental distribution network with high penetration of distributed photovoltaic (Day-ahead optimal scheduling method for incremental distribution network with high penetration of distributed photovoltaic) $[\mathrm{J}]$. Power System Protection and Control (Power System Protection and Control), 2019, 47(18):90-98.

4. Ding Ming, Fang Hui, Bi rui, et al. (Ding Ming, Fang Hui, Bi rui, et al.). Optimal siting and sizing of distributed PV-storage in distribution network Based on cluster partition [J]. Proceedings of the CSEE, 2019, 39(08): 2187-2201+2..

5. Huang Chunyi, Wang Chengmin, Xie Ning, et al. (Huang Chunyi, Wang Chengmin, Xie Ning, et al.). Distribution expansion planning based on strong coupling of operation and spot market[J]. Proceedings of the CSEE, 2019, 39(16): 4716$4731+4974$.

6. Yang J, Gao H, Ye S, et al. Applying multiple types of demand response to optimal day-ahead stochastic scheduling in the distribution network $[\mathrm{J}]$. IET Generation, Transmission \& Distribution, 2020.

7. Zhang Muyin, Zhou Ling, Guan Zhicheng, et al. (Zhang Muyin, Zhou Ling, Guan Zhicheng, et al.). Multi-objective coordination planning of distribution network with distributed generation considering the impact of demand response (Multiobjective) coordinated planning of distribution network with distributed generation considering influence of demand response)[J].Advanced Technology of Electrical Engineering and Energy, 2017, 36(03): 16-21 